\title{
Harnessing molecular rotations in plastic crystals: a holistic view for crystal engineering of adaptive soft materials
}

\author{
Susobhan Das, Amit Mondal and C. Malla Reddy* \\ Department of Chemical Sciences, Indian Institute of Science Education and Research (IISER) Kolkata, Mohanpur Campus, \\ Mohanpur, West Bengal, 741246, India
}

dassusobhan.chem@gmail.com; cmallareddy@gmail.com

Abstract: Plastic crystals (PCs), formed by certain types of molecules or ions with reorientational freedom, offer both exceptional mechanical plasticity and long range order, ${ }^{[1,2]}$ hence they are attractive for many mechano-adaptable technologies. While most classic PCs belong to simple globular molecular systems, a vast number of examples in the literature with diverse geometrical (cylindrical, bent, disk, etc.) and chemical (neutral, ionic, etc.) natures have proven their wide scope and opportunities. ${ }^{[2,3]}$ All the recent reviews on PCs aim to provide insights into a particular application, for instance, organic plastic crystal electrolytes or ferroelectrics. ${ }^{[4-6]}$ This tutorial review presents a holistic view of PCs by unifying the recent excellent progress in fundamental concepts from diverse areas as well as comparing them with liquid crystals, amphidynamic crystals, ordered crystals, etc. ${ }^{[7]} \mathrm{We}$ cover the molecular and structural origins of the unique characteristics of PCs, such as exceptional plasticity, facile reversible switching of order-to-disorder states and associated colossal heat changes, and diffusion of ions/molecules, and their attractive applications in solid electrolytes, optoelectronics, ferroeletrics, piezoelectrics, pyroelectrics, barocalorics, magnetics, nonlinear optics, and so on. ${ }^{[6,7,9]}$ The recent progress not only demonstrates the diversity of scientific areas in which PCs are gaining attention but also the opportunities one can exploit using a crystal engineering approach, for example, the design of novel dynamic functional soft materials for future use in flexible devices or soft-robotic machines. ${ }^{[3,7,9,10]}$
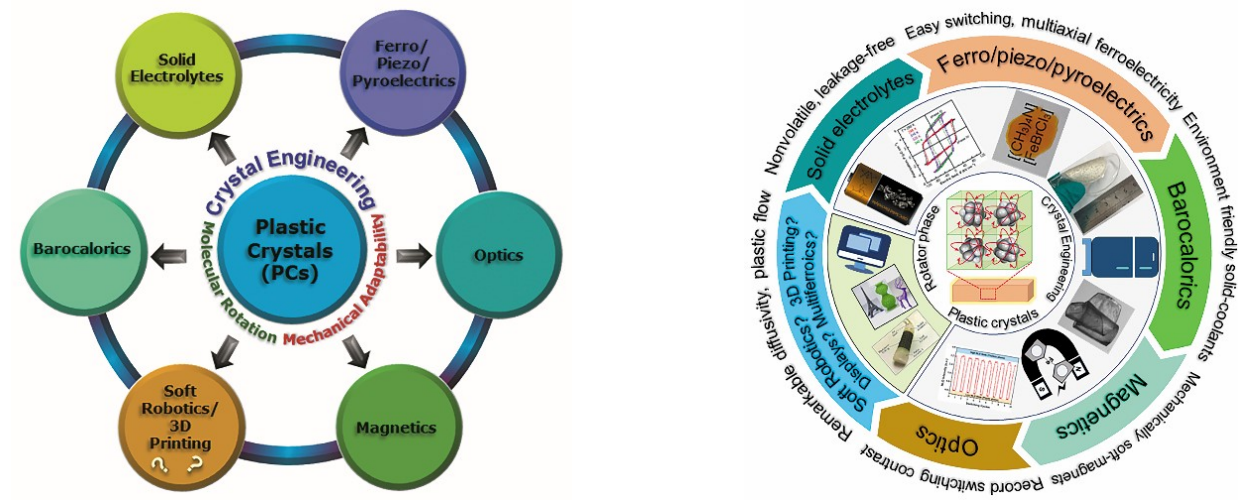

Figure 1. General overview of areas of prominence for PCs (left) and their expanding scope in material sciences with plenty of opportunities (right).

[1] Timmermans, J. (1961). J. Phys. Chem. Solids, 18, 1.

[2] Sherwood, J. N. (1979). The Plastically Crystalline State: Orientationally Disordered Crystals, John Wiley \& Sons Ltd, New York.

[3] Mondal, A., Bhattacharya, B., Das, S., Bhunia, S., Chowdhury, R., Dey, S. \& Reddy, C. M. (2020). Angew. Chem., Int. Ed., $59,2$.

[4] Zhu, H., MacFarlane, D. R., Pringle, J. M. \& Forsyth, M. (2019). Trends Chem., 1, 126.

[5] Luo, J., Jensen, A. H., Brooks, N. R., Sniekers, J., Knipper, M., Aili, D., Li, Q., Vanroy, B., Wubbenhorst, M., Yan, F., Meervelt, L. V., Shao, Z., Fang, J., Luo, Z.-H., De Vos, D. E., Binnemans K. \& Fransaer, J. (2015). Energy Environ. Sci., 8, 1276.

[6] Tang, Y. Y., Li, P. F., Liao, W. Q., Shi, P. P. You, Y. M. \& Xiong, R. G. (2018). J. Am. Chem. Soc., 140, 8051.

[7] Das, S., Mondal, A. \& Reddy, C. M. Chem. (2020). Soc. Rev., 49, 8878.

[8] Zhang, Y., Song, X.-J., Zhang, Z.-X., Fu D.-W. \& Xiong, R.-G. (2020). Matter, $2,697$.

[9] Li, B., Kawakita, Y., Kawamura, S. O., Sugahara, T., Wang, H., Wang, J., Chen, Y., Kawaguchi, S. I., Kawaguchi, S., Ohara, K., Li, K., Yu, D., Mole, R., Hattori, T., Kikuchi, T., Yano, S.-I., Zhang, Z., Zhang, Z., Ren, W., Lin, S., Sakata, O., Nakajima, K. \& Zhang, Z. (2019). Nature, $567,506$.

[10] Saha, S., Mishra, M. K., Reddy, C. M. \& Desiraju, G. R. (2018). Acc. Chem. Res., 51, 2957.

Keywords: Plastic crystals; Molecular rotation; Crystal engineering; Adaptable electronics; Mechanical properties Acta Cryst. (2021), A77, C1017 